\title{
$\checkmark$ Research Square \\ Radar-Based Timed Up And Go Test For Multiperson Providing A Risk Of Falling Assessment
}

Johannes C. Ayena

Ecole de technologie superieure

Lydia Chioukh ( $\nabla$ chioukh.lydia@gmail.com )

Ecole de technologie superieure

Dominic Deslandes

Ecole de technologie superieure

\section{Research Article}

Keywords: Falls, TUG, UWB radar, TUG, UWB radar, gait parameters, non-contact.

Posted Date: March 7th, 2022

DOI: https://doi.org/10.21203/rs.3.rs-1282942/v1

License: (c) (i) This work is licensed under a Creative Commons Attribution 4.0 International License.

Read Full License 


\title{
Radar-based timed up and go test for multiperson providing a risk of falling assessment
}

\author{
Johannes C. Ayena, Lydia Chioukh and Dominic Deslandes* \\ Communications and Microelectronic Integration Laboratory (LACIME), Department of Electrical \\ Engineering, École de Technologie Supérieure, 1100 Rue Notre-Dame Ouest, H3C1K3, Montréal, \\ Qc., Canada \\ Email: cossoun-johannes.ayena.1@ens.etsmtl.ca; lydia.chioukh.1@ens.etsmtl.ca \\ *Correspondence: dominic.deslandes@etsmtl.ca
}

\begin{abstract}
Objective: Over the past decade, the research community has made major advances in technologies for detecting risk of falling in elderly, which led to assistive systems that can help to improve physical strength and limit isolation. However, it is still expensive to extend these systems to a multi-user performing a clinical test, such as the Timed Up and Go (TUG). In this paper, we therefore propose a new avenue, a radar-based system that transcends these limitations.

Results: The proposed methodology is evaluated using data from real-world scenarios. It is found that distinguishing two participants performing a TUG test at the same time is more accurate when they are walking in opposite direction compared to perfectly synchronized walking in the same direction. However, although it was more challenging to distinguish two participants in this latter scenario, our results show that when they are offset by a certain distance, the proposed algorithm can track them separately. Our goal was therefore to relieve congestion in medical settings by investigating the feasibility of administering a clinical test with more than one participant at the same time thanks to an UWB radar sensor.
\end{abstract}

Keywords: Falls; TUG; UWB radar; TUG; UWB radar; gait parameters; non-contact. 


\section{Introduction}

Elderly patients with COVID-19 or other types of viruses are rapidly progressing to more serious health conditions and have significantly higher mortality rates than younger patients [1]. Thus, as a preventive measure, public health in each country of the world is implementing measures of containment and social distancing. This reduction in social interaction leads to an increase in functional limitations and expands not only the risk of falling, the risk of aggravating a chronic disease but also the risk of mortality [2]. To address these realities from isolation and physical inactivity, several methods and devices are offered to these individuals at risk [3-5]. Banskota et al. [6] have summarized 15 smartphone apps which can be used to improve older adult's quality of life, especially during social distancing or self-quarantine. Although, apps and such technologies can be inexpensive and accessible, not all older people are able to use them properly, even if they have received the necessary training.

Recently, several nursing homes have been allowed to let family caregivers back in their facility $[7,8]$. However, despite this help, maintaining mobility in old age is necessary, as it may predict falls in older adults. Indeed, physical inactivity in older people is the fourth highest risk factor for mortality worldwide and contribute to disability [9]. Radar technology can be exploited, as it offers a continuous monitoring without direct contact in the user's skin. Compared to the current available devices in the literature [10], radar system transforms the way in which we needed to interact with the elderlies.

In this line of thoughts, several studies [11-13] are devoted to track multi-person performing static and dynamics movements. However, most of them involve random activities which are not adequate for training an elderly. In fact, most of the suggested methods are not directly applicable to sequential activities such as sit-to-stand and walking, walking and turning, etc., which better highlight gait and balance impairments need to be trained. We are therefore investigating the possibility of using multiperson tracking techniques combined with the realization of a clinical test such as a Timed Up and Go (TUG) test dedicated to enhance balance performance among older adults. Indeed, multi-person tracking is increasingly becoming important in many applications. As summarized and compared in [14], random finite set (RFS) multi-target tracking algorithms such as the probability hypothesis density (PHD) filter has received a great attention. The PHD filter [15] is the most widely RFS based filter used for visualizing the tracking of multiple targets. His advantage is its ability to track a variable number of participants, estimating both the number of participants and their locations. It has better tracking performance comparing to Kalman Filter (KF) in the presence of clutter [16]. In this study, we use Gaussian Mixture (GM) PHD.

The contribution of this research is to identify the trajectories of different participants who are performing a TUG test. For doing so, we improved the PHD filter by adding a data association technique, the global nearest neighbor (GNN), to better identify separately the multiple participants involved in the clinical test. It is a simple method that can be implemented easily in real time. This approach attempts to find a predefined number of samples closest in distance to the new point and propagate the single most likely hypothesis at each scan. The procedure of this approach is described in [17]. The main goal of this article is to develop a first system that could track the trajectories of multi persons moving with a clear difference using a single device. 


\section{Main text}

\section{Materials and Methods}

\section{Proposed rTUG test}

Contrary to previous studies, we propose here the possibility of performing the test TUG with more than one person at once (Fig.1) using an UWB radar (Xethru X4M200). All information about architecture and functionalities of this radar system are shown in $[18,19]$. This new proposed system has been evaluated in different test scenarios in home setting with two participants, the authors of this manuscript. In each scenario, the participants were seated on its chair separated by $1.2 \mathrm{~m}$ (as shown in Fig.1). We asked them to stand up from their respective chair, walking separately in a $3 \mathrm{~m}$ corridor, turn 180 deg., and return to sit in their respective chair, except for scenario 1, in which one of the participants stays seated.

\section{Data analysis}

We hypothesized that the PHD filter filtered out most false alarms so that all estimated values in every step $(k)$ are considered as targets, and that there is only one association per target.

For each of the multiple outputs $\left(\left\{Z_{k, 1}, \ldots . . Z_{k, N_{k}}\right\}\right)$ related to the position and velocity, and estimated by the PHD filter at time $k$, we create a validation region as follows:

$$
V_{k, i}:\left\{z:\left[Z_{k, i}-Z_{k}^{p}\right]^{T}\left(S_{k, i}\right)^{-1}\left[Z_{k, i}-Z_{k}^{p}\right] \leq G\right\}
$$

where the predicted measurement $Z_{k}^{p}\left(Z_{k}^{p}=F Z_{k-1}, j\right)$ is obtained by projecting the previous estimate $Z_{k-1}, j$ using the motion model $F$ i.e., the state transition matrix containing constant values. $G$ is the validation gate:

The index $i$ represents one of the outputs of the PHD filter at a time whereas the index $j$ represents the last measure representing the tracking of the targets. $S_{k}=H P_{k \mid k-1} H^{T}+R$ is the innovation covariance. The predicted estimate covariance is equal to:

$$
P_{k \mid k-1}=F P_{k-1 \mid k-1} F^{T}+Q_{k}
$$

where $H$ is the observation matrix with constant values, $Q_{k}$ is the process noise covariance, $F^{T}$ is the transpose of the transition matrix and $R$ the observation noise covariance. 
S2: The two participants start moving from the

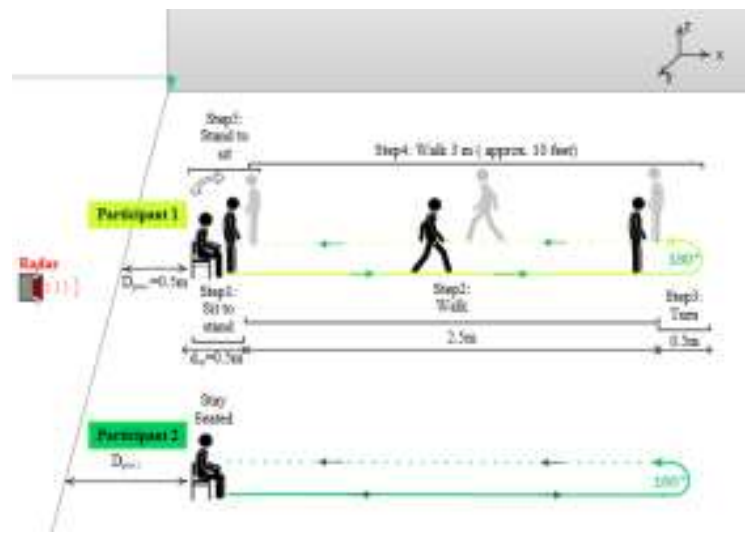
same position for the same direction

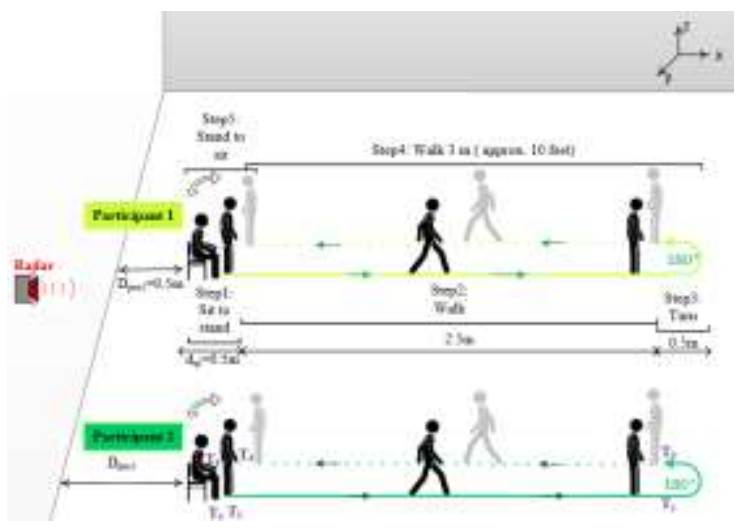

S3: The two participants start moving from different position for the same direction

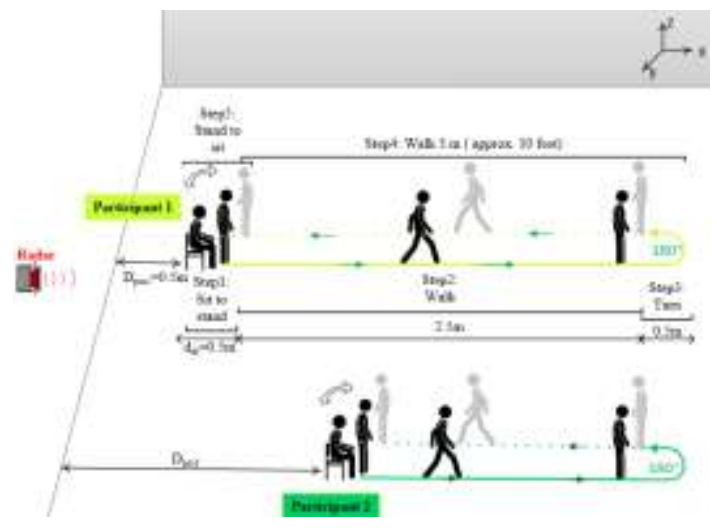

S4: The two participants start moving from different position and opposite direction

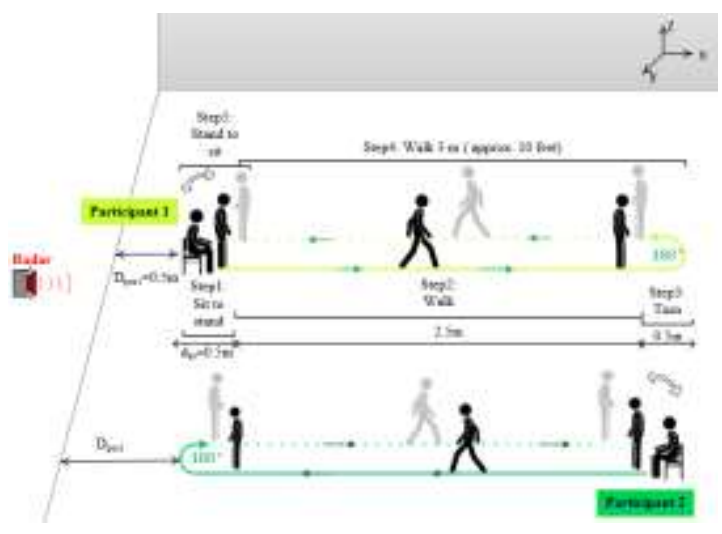

Fig. 1 Different scenarios for testing the proposed rTUG system

Dos: the distance between the location of the radar and that of the chair; $d_{q s}$ : the distance between the chair and the person in standing position which correspond to the sit-to-stand activity).

The validation gate $G$ presented in Eq. 1 is obtained from the inverse chi-square cumulative distribution [20] so that the validation region was large enough to include the true measurement corresponding to each participant. Our proposed algorithm (Fig.2) combines the measurements from the previous time by deciding if:

1) the current value of the target can update one of the available tracks (the measurement is valid and it lies inside the elliptical validation gate),

2) the target is dead at the time $k$ or 
3) the target can be declared as a newborn (no association has been made and are defined as new tracks). Those are followed during a short period and updated in order to keep it as being real targets or not. If, during a period of $1 \mathrm{~s}$, the new states of the targets cannot update the new tracks, this new track is removed. We assume that a new target cannot enter the radar field of view (FOV) and leave it at the same time within 1s. Thus, if any new track can be updated by the new states during a period of more than $3 \mathrm{~s}$, this should be considered as a real new target in the FOV of the radar.

\section{Results}

Fig.3 presents four scenarios for evaluating at the same time two participants during a TUG test. In the first scenario (S1), one participant is seated, and the second participant is moving (Fig.3a). In S2, the two participants start from the position $D_{\text {pos }}=0.5 \mathrm{~m}$ and walk in a straight line until $3 \mathrm{~m}$. Then, they turn $180^{\circ}$ and walk back (Fig.3b). The participants walk with their normal speed. In Fig.3, the blue line is the original data of the targets path which are from the radar, the red line is the estimates of the PHD filter algorithm only, and the black line is the estimates of our proposed algorithm.

In Figs.3a and 3b, no difference is found between these two scenarios. This means that if two persons are perfectly synchronized and the radar is placed exactly in the middle between the persons, the radar considers them as a single object. However, we can clearly see the differences between the black line and the red line in this part of the experiments, which indicates that the positions of the person can be estimated accurately during a TUG test by our proposed algorithm. Indeed, determining the position of an elderly performing a clinical test is important insofar as it will allow to know rather if a falling occurs.

The second part of the experiments (S3 and S4) involves a different target path (Figs. 3c and 3d). In S3, the first person starts from the position $D_{\text {pos } 1}$ and the other person starts from the position $D_{\text {pos2 }}$. The black and magenta lines represent the outputs of our proposed algorithm. Thus, for these scenarios, the algorithm performs well. There are some differences between the first and the second part of the scenarios. For instance, the radar sensor measurements are more challenging with the multiple person case, which can be observed by comparing the sensor data in Fig. 3a with that in Fig. 3c. One of the more difficult monitoring scenarios is the one illustrated in Fig.3b. When the targets 
walk side by side and the radar is centered, they cannot be detected separately. However, our proposed algorithm still provides an accurate tracking in this challenging scenario.

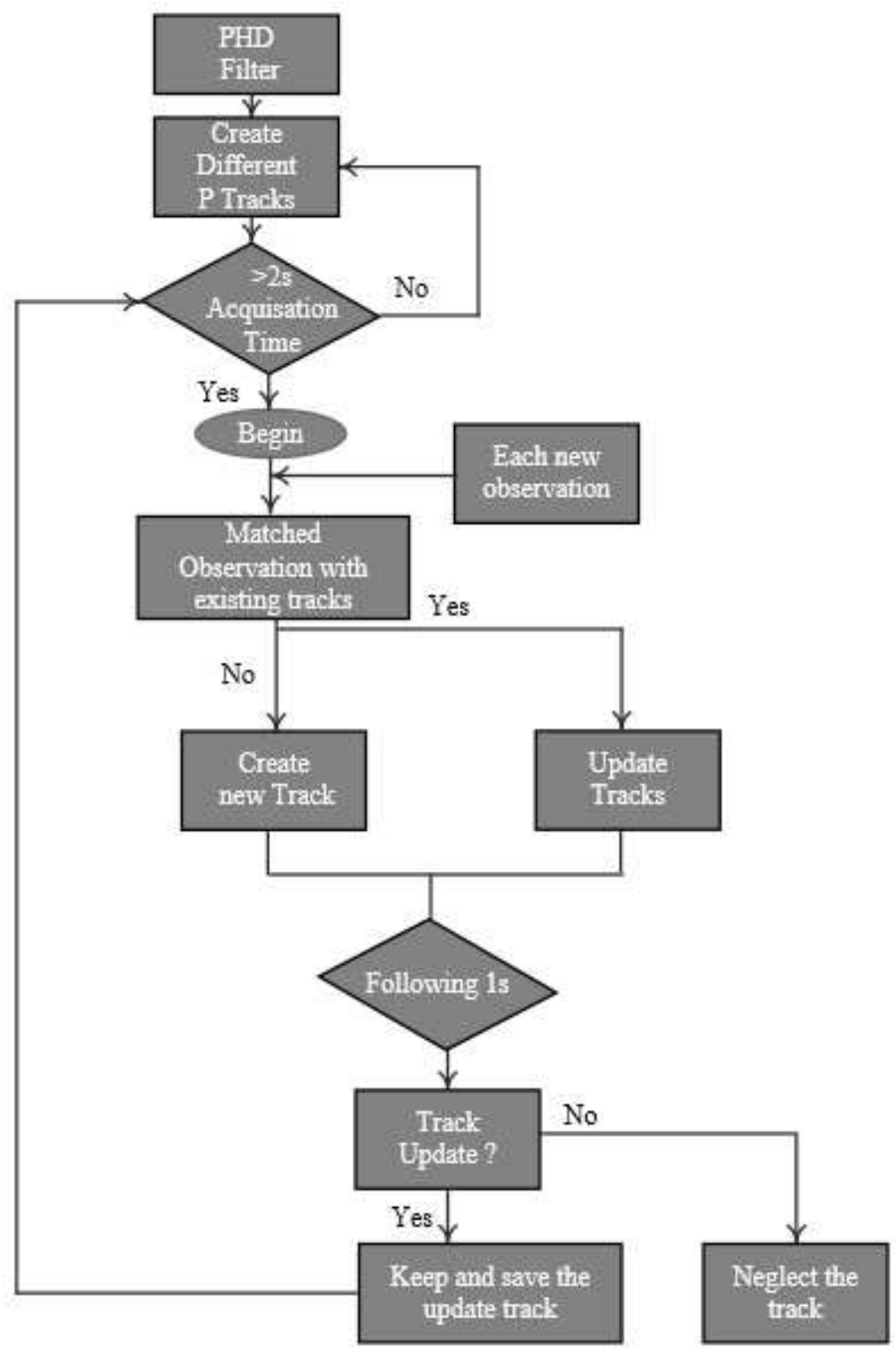

Fig. 2 The flow diagram of the automated algorithm for detecting and tracking each participant involved in the TUG test using the UWB radar. 

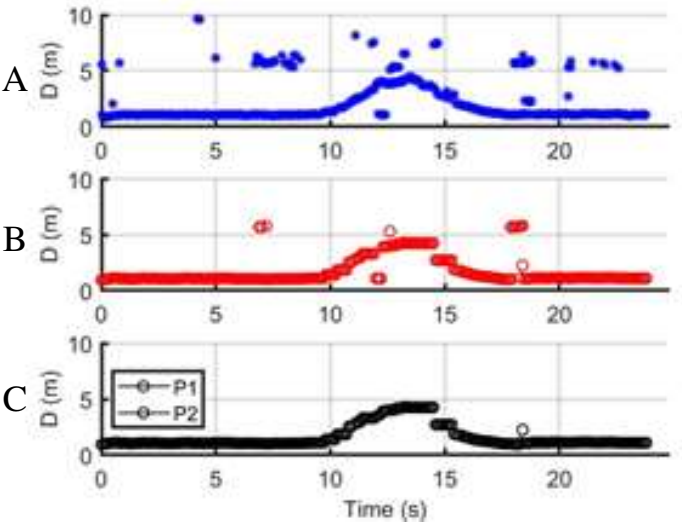

(a)

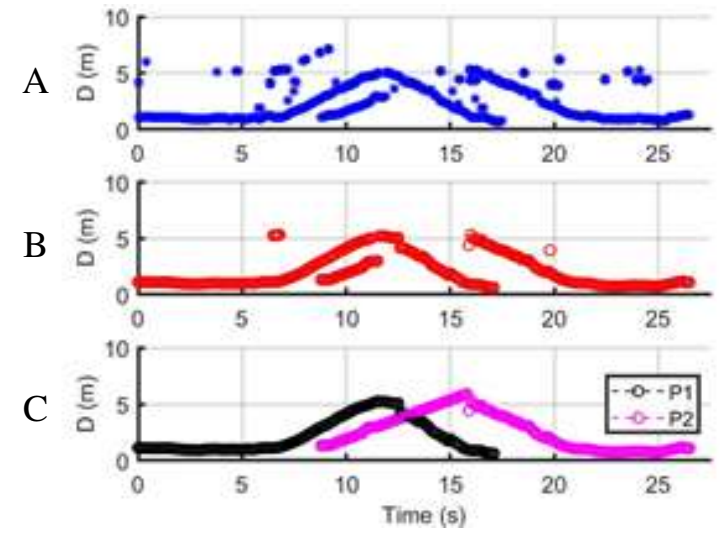

(c)
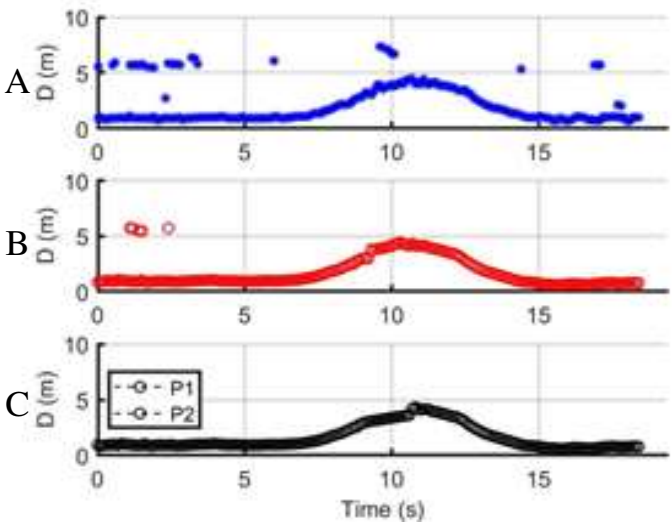

(b)

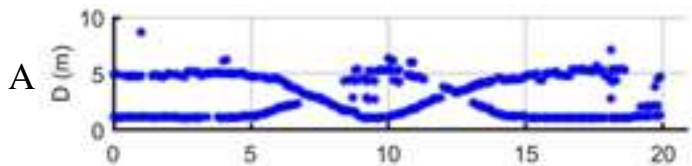

B

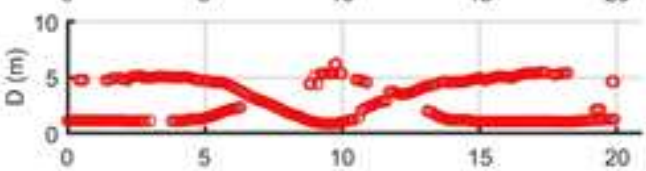

$\mathrm{C}$

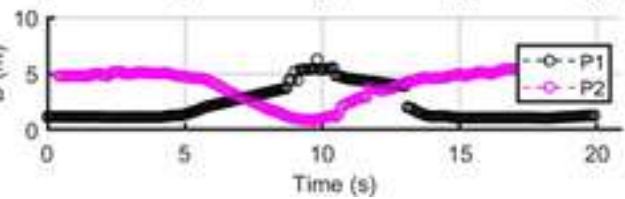

(d)

Fig. 3 Identifying two participants (P1 and P2) separately during a TUG test for different scenarios corresponding respectively to S1, S2, S3 and S4 (radar tracking the two participants).

Note: (A): The blue curve represents the data collected directly from the radar (the original data); (B): The red curve represents the PHD filter (PHD only) applied on the raw data; (C): The black and pink curves represent our proposed algorithm (the combination of the PHD filter and the GNN algorithm)

\section{Discussion}

Across all the four scenarios, our outcomes are in the line of the studies suggested in [11]. An interesting finding in our experiments was that, rather than testing one participant, two participants can be tested at the same time. Many studies [21,22] often involve spouses or kinships as control participants when testing the participants with gait impairments. So, we think that our proposed methodology can improve efficiency the way these tests are given.

In radar applications, successive missed detections can occur due to sensor failure, low probability of detection, or when targets become obstructed. Many previous studies did not investigate possible 
subsequent missed detections that may occur in practical applications. In this paper, another aspect of our contribution was then to improve the PHD filter that attempts to recover the subsequent missed detections as much as possible (Fig. 2). To achieve this goal, we introduce the GNN algorithm on the targets estimated by the PHD filter. This algorithm provides some advantages when failure detection occurs in subsequent frames. The results of our proposed method not only outperform the techniques in these fields but also enable a fully automated TUG sub-task segmentation to be performed using the algorithm described in [19]. Without a wearable device technology, we believe that the duration of the TUG sub-tasks using a radar can also be exploited to provide information on the risk of falling, where the elderlies take more time during the test, the motor characteristics, etc. [23-25]. As shown in Fig.3, we can see that the GNN method have a clear impact on the performance of the PHD filter. Since mis-association should be avoided as much as possible, we therefore investigated different scenarios as presented (Fig.1). Indeed, we are confident that the clear differences found between the two participants (Figs.3c and 3d) cannot be attributed to a hazardous design of scenarios. Since the PHD filter can track multiple targets at the same time $[15,26]$, we believe that the proposed design scenarios are appropriate enough to motivate an elderly person to perform physical activity regularly.

\section{Conclusions}

The main goal of this pilot study is to relieve congestion in medical settings by investigating the feasibility of administering a TUG test with more than one participant at the same time thanks to an UWB radar sensor. For doing so, the proposed system tracks the trajectories of moving persons and we had investigated the main contribution in different aspects and scenarios. Our findings showed that a single radar placed at a distance $d$ behind a chair may be used to automate the TUG test in multiple participants thanks to PHD filter. The UWB device will be used for more extensive testing of multiperson and other emerging applications.

\section{Limitations}

The limitation of this study is the generalization of finding to a wider population, due to the small sample size used. However, this was a preliminary study as proof of concepts. Thus, more research should confirm our findings in larger groups. Thresholds for gate validation are currently defined and 
based on experiences. In the future, we want to investigate these thresholds according to more participants' individual capabilities to make the computation more reliable in larger sets of participants.

\section{Declarations}

\section{Abbreviations}

COVID: coronavirus disease, GM: Gaussian mixture, GNN: Global nearest neighbor, KF: Kalman filter, TUG: Timed up and go test, UWB: Ultra-wide band

\section{Acknowledgements}

We would like to thank Sid Ali Garidi for all given support to the authors.

\section{Author's contributions}

JCA developed the methods and software and generated the figures with inputs from LC. LC designed the research project. JCA and LC analyzed the data, drafted the manuscript under the supervision of DD. All authors interpreted the results and substantially revised the draft. All authors read and approved the final manuscript

\section{Availability of data and material}

Not applicable

\section{Competing interests}

The authors declare that they have no competing interests

\section{Conflicts of Interest}

The authors declare no conflict of interest and the funders had no role in the design of the study; in the collection, analyses, or interpretation of data; in the writing of the manuscript, or in the decision to publish the results".

\section{Consent for publication}

Not applicable

\section{Ethics approval and consent to participate}

Not applicable

\section{Funding}

This research was funded by the Natural Sciences and Engineering Research Council of Canada (NSERC). 


\section{References}

1. Liu K, Chen Y, Lin R, Han K: Clinical features of COVID-19 in elderly patients: A comparison with young and middle-aged patients. J Infect 2020, 80(6):e14-e18.

2. Brooke J, Jackson D: Older people and COVID-19: Isolation, risk and ageism. J Clin Nurs 2020, 29(1314):2044-2046.

3. Gorenko JA, Moran C, Flynn M, Dobson K, Konnert C: Social Isolation and Psychological Distress Among Older Adults Related to COVID-19: A Narrative Review of Remotely-Delivered Interventions and Recommendations. Journal of Applied Gerontology 2020, 40(1):3-13.

4. Campos W, Martinez A, Sanchez W, Estrada H, Castro-Sánchez NA, Mujica D: A Systematic Review of Proposals for the Social Integration of Elderly People Using Ambient Intelligence and Social Networking Sites. Cognitive Computation 2016, 8(3):529-542.

5. Embarak F, Ismail NA, Othman S: A systematic literature review: the role of assistive technology in supporting elderly social interaction with their online community. Journal of Ambient Intelligence and Humanized Computing 2021, 12(7):7427-7440.

6. Banskota S, Healy M, Goldberg EM: 15 Smartphone Apps for Older Adults to Use While in Isolation During the COVID-19 Pandemic. West J Emerg Med 2020, 21(3):514-525.

7. Verbeek H, Gerritsen DL, Backhaus R, de Boer BS, Koopmans RTCM, Hamers JPH: Allowing Visitors Back in the Nursing Home During the COVID-19 Crisis: A Dutch National Study Into First Experiences and Impact on Well-Being. Journal of the American Medical Directors Association 2020, 21(7):900-904.

8. Bergman C, Stall NM, Haimowitz D, Aronson L, Lynn J, Steinberg K, Wasserman M: Recommendations for Welcoming Back Nursing Home Visitors During the COVID-19 Pandemic: Results of a Delphi Panel. Journal of the American Medical Directors Association 2020, 21(12):1759-1766.

9. Goethals L, Barth N, Guyot J, Hupin D, Celarier T, Bongue B: Impact of Home Quarantine on Physical Activity Among Older Adults Living at Home During the COVID-19 Pandemic: Qualitative Interview Study. JMIR Aging 2020, 3(1):e19007.

10. Sun R, Sosnoff JJ: Novel sensing technology in fall risk assessment in older adults: a systematic review. BMC Geriatrics 2018, 18(1):14.

11. B. Gulmezoglu, M. B. Guldogan, Gezici S: Multiperson tracking with a network of ultrawideband radar sensors based on gaussian mixture PHD filters. IEEE Sensors Journal Apr. 2015, 15(4):2227-2237.

12. Zhang D, Hu Y, Chen Y: MTrack: Tracking Multiperson Moving Trajectories and Vital Signs With Radio Signals. IEEE Internet of Things Journal 2021, 8:3904-3914.

13. Canton-Ferrer C, Salvador J, Casas JR, Pardàs M: Multi-person Tracking Strategies Based on Voxel Analysis. In: Multimodal Technologies for Perception of Humans: 2008// 2008; Berlin, Heidelberg: Springer Berlin Heidelberg; 2008: 91-103.

14. Pulford GW: Taxonomy of multiple target tracking methods. IEE Proceedings - Radar, Sonar and Navigation 2005, 152(5):291-304.

15. Vo B, Ma W: The Gaussian Mixture Probability Hypothesis Density Filter. IEEE Transactions on Signal Processing 2006, 54(11):4091-4104.

16. Nabil M, Kamal H, Hassan M: Comparison between Kalman Filter and PHD Filter in Multi-target Tracking. The International Conference on Electrical Engineering 2012, 8(8th International Conference on Electrical Engineering ICEENG 2012):1-14. 
17. Konstantinova PD, Udvarev A, Semerdjiev T: A study of a target tracking algorithm using global nearest neighbor approach. In: CompSysTech '03: 2003; 2003.

18. $\mathrm{X} 4 \mathrm{~m} 200$

datasheets

[https://www.codico.com/shop/media/datasheets/Novelda X4M200 respiration sensor rev c preliminary .pdf]

19. Ayena JC, Chioukh L, Otis MJ-D, Deslandes D: Risk of Falling in a Timed Up and Go Test Using an UWB Radar and an Instrumented Insole. Sensors 2021, 21(3):722.

20. Sualeh M, Kim G-W: Dynamic Multi-LiDAR Based Multiple Object Detection and Tracking. Sensors 2019, 19(6):1474.

21. Ponciano V, Pires IM, Ribeiro FR, Marques G, Garcia NM, Pombo N, Spinsante S, Zdravevski E: Is The Timed-Up and Go Test Feasible in Mobile Devices? A Systematic Review. Electronics 2020, 9(3):528.

22. Ayena JC, Tremblay LE, Otis MJD, Ménélas B-AJ: Comparing auditory, visual and vibrotactile cues in individuals with Parkinson's disease for reducing risk of falling over different types of soil. Somatosensory $\mathcal{E}$ Motor Research 2017, 34(4):226-234.

23. Kurosawa C, Shimazu N, Yamamoto S: Where do healthy older adults take more time during the Timed Up and Go test? J Phys Ther Sci 2020, 32(10):663-668.

24. Hsieh C-Y, Huang H-Y, Liu K-C, Chen K-H, Hsu SJ-P, Chan C-T: Subtask Segmentation of Timed Up and Go Test for Mobility Assessment of Perioperative Total Knee Arthroplasty. Sensors 2020, 20(21):6302.

25. Mangano GRA, Valle MS, Casabona A, Vagnini A, Cioni M: Age-Related Changes in Mobility Evaluated by the Timed Up and Go Test Instrumented through a Single Sensor. Sensors (Basel) 2020, 20(3):719.

26. Li Y, Guo L, Huang X: Research and Application of Multi-Target Tracking Based on GM-PHD Filter. Optics and Photonics Journal 2020, Vol.10No.06:9. 